\title{
Development of the health-preserving competence of a physical education teacher based on the knowledge about influenza and bronchitis prevention
}

\author{
Vasyl M. Fedorets ${ }^{1, *}$, Mykola B. Yevtuch ${ }^{2}$, Oksana V. Klochko ${ }^{3, * *}$, Nina P. Kravets ${ }^{4}$, and Roman S. Grynyov ${ }^{5}$ \\ ${ }^{1}$ Department of Psychological and Pedagogical Education and Social Sciences, Public higher educational establishment "Vinnytsia \\ academy of continuing education", 13 Hrushevskoho Str., Vinnytsia, 21000, Ukraine \\ ${ }^{2}$ Institute of Pedagogy of the National Academy of Educational Sciences of Ukraine, 52-D Sichovykl Striltsiv Str., Kyiv, 04053, Ukraine \\ ${ }^{3}$ Department of Mathematics and Informatics, Vinnytsia Mykhailo Kotsiubynskyi State Pedagogical University, 32 Ostrozkoho Str., \\ Vinnytsia, 21001, Ukraine \\ ${ }^{4}$ Department of Psychocorrection Pedagogy, National Pedagogical Dragomanov University, 9 Pyrohova Str., Kyiv, 01601, Ukraine \\ ${ }^{5}$ Physics Department, Faculty of Natural Sciences, Ariel University, 65 Ramat HaGolan Str., Ariel, 40700, Israel
}

\begin{abstract}
The article presents the methodology and methods of improving the health-preserving competence of a Physical Education teacher under conditions of postgraduate education based on the knowledge of prevention of acute bronchitis, COVID-19 influenza and acute respiratory diseases. The "Disciplinary and methodological matrix of health-preserving competence of a Physical Education teacher" is used, which includes such developed "integrated disciplines" as Pathopedagogy, Propaedeutics of Health and Health Therapy. The matrix is aimed at professionalisation, methodologisation, technologisation, anthropologisation, axiologisation of the health-preserving competence of a teacher. The "Methodology of developing health-preserving competence of a Physical Education teacher based on the knowledge of prevention of acute bronchitis, influenza and acute respiratory diseases" is used. The methodology is based on the competence approach, the above-mentioned matrix, problem-based and flipped learning, andragogy, game, maieutic and dialogic methods, the use of pedagogical tasks, the Hellenistic concept of "self-care" and others. In this methodology, knowledge, values and practical health-preserving strategies are hierarchised with the singling out of three levels - A, B and C. Level $\mathrm{A}$ is obligatory, and levels B and C are given less attention. An experimental study was conducted the result of which determined the effectiveness of this method. These methodology and method are humanistic and ecocentric and consistent with the ideas and sustainable development goals.
\end{abstract}

\section{Introduction}

The health-preserving competence of a Physical Education teacher [1] is a professional and personal as well as epistemological and value professional "toolkit", which is aimed primarily at the optimal solution by a teacher of specific problems, tasks and situations connected with both physical activity and children's health. That is, in the professional activity of a Physical Education teacher, the complexity and multidimensionality of human nature are revealed, first of all, in the format of working with practically significant phenomena. In the professional "reality" of an educator, the above-mentioned phenomena are revealed as a system of relevant and significant, practical and technological issues. For the optimal solution of such issues, a teacher requires intellectual skills in making specific (in the sense of concretised and adapted to a problem), practical and life creation-oriented optimal decisions, as well as the presence of special knowledge and practical skills to implement strategies and tactics related to certain problems.

\footnotetext{
*e-mail: bruney333@yahoo.com

**e-mail: klochkoob@gmail.com
}

Nowadays the existing tradition of decision-making in complex pedagogical situations involving risks to health and life is based primarily on the prescription "format" of knowledge presented mainly in the form of certain recommendations. The issue of the "prescription" nature of the application concerns the educational range of problems of health-preserving technologies, strategies and practices. In this aspect, it is the range of problems of their development, selection, specifics of use and strategic issues of the necessity and expediency of implementation that are relevant. The above-mentioned "prescription" tradition is certainly optimal, but at the same time insufficient for many reasons, namely: not full disclosure of human phenomenology under conditions of physical exercise and pathology, by the fact that the recommendations reflect not only the leading ideas of scientific schools but also cultural traditions and stereotypes, which may not be sufficiently objective and relevant to reality; by unformed and undisclosed appreciative and semantic "reality" and a motivational factor as those that determine personal and activity prerequisites of practices and technologies. That is, such recommendations are not always sufficiently objective, value and activity-oriented, scientific, evidential and, 
in general, inconsistent with the competence paradigm. In this aspect of the range of problems, the dominance of general recommendations for preserving health has great importance as well as the predominance of healthy lifestyle traditions, in which the phenomenology of a human both under normal conditions and, first of all, under conditions of pathology are not taken into account in full.

Based on the ideas of evidential nature and guided by the intentions of scientific nature, it can be noted that nowadays the existing ideology of child-centeredness $[2,3]$ and humanism [4] in education determine the need to form the teacher's ability to make optimal health-oriented decisions. Accordingly, such decisions must be "intellectualised", "anthropologised" [5, 6] (that is, formed on the basis of knowledge of human nature), axiologised and evidential from the standpoint of both science and practice. Such anthropologised decisions must be based primarily on the transfer of not only special medical and hygienic, valeological and anthropological knowledge [5, 6], but also be realised by way of the reception of appropriate algorithms of thinking, cognitive schemes, values, some components of practices and technologies, professional ethics and stereotypes of behaviour.

It should be noted that in our time the potential of anthropological knowledge, which is necessary for the development and use in educational technologies and healthpreserving practices, including the ideas of pathology, is not used in full. That is, there is a methodologically and practically significant discrepancy between the existing significant level of anthropobiological and, in general, anthropological knowledge and their insufficient use in pedagogy and education. Targeted and problem-oriented selection and, accordingly, practice-oriented actualisation and representation of that knowledge of a human (child) which are necessary for the analysis and resolving typical problems and situations, in which the nature of a human is reflected, are important for effective preservation of health under conditions of an educational process. Accordingly, the technological and value-orientation as well as specification (in the sense of concretisation) of knowledge (both general and specialist) about a human (child) both under normal conditions and under conditions of pathology are also important.

Therefore, phenomenology- and problem-oriented understanding of the professional reality of a Physical Education teacher, we emphasise the need to update and disclose specific practically significant phenomena, with which he/she works in the process of improving the level of professional skills. Such a life-creative and practiceoriented approach is also in line with the spirit and meanings of the competency and innovative paradigms. Accordingly, we turn to the need for practice-oriented disclosure of educationally and vitally important range of problems of acute bronchitis prevention [7, 8]. This pathology (in the sense of a disease) - acute bronchitis might be the result of "ordinary" influenza, Covid-19 [9], acute respiratory infection, hypothermia and stress situation. Acute bronchitis is a phenomenon that occurs quite often. At the same time, acute bronchitis [10] as a specific educational and pedagogical problem is not given enough atten- tion. Therefore, we actualise, as an educationally significant problem, the preservation of health of students under condition of an educational process based on the coverage of the phenomenology of respiration and respiratory system in relation to the transfer of knowledge of prevention of acute bronchitis, as well as by studying the possibility of correcting the consequences of this pathology with the help of physical culture means. The above-mentioned range of problems is considered by us in innovative and systematic aspects. Technologically value understanding of the phenomenon of acute bronchitis is significant in terms of the development of health-preserving thinking of a Physical Education teacher and the formation of his/her professional aims, attitudes, intentions and values.

Now a central system-organizing and key aspect, which highlights the relevance and significance of this problem, is the Covid-19 pandemic [11-15]. A special life-saving aspect, which reveals the vital importance of this range of problems, is the issues of prevention of complications of acute bronchitis [10] under conditions of an educational process, including the physical activity of students during Physical Education lessons. Such complications include life-threatening pneumonia as well as chronic bronchitis and bronchial asthma [7, 8]. In the context of actualisation of this problem, it is important to realise that a Physical Education teacher does not really work with an "ideal" child who is "always" healthy. $\mathrm{He} / \mathrm{she}$ runs classes with children who have certain peculiarities and pathologies which correspond to human nature. Therefore, the above-mentioned peculiarities of those "real" children and systematic risks, which are "dictated" by human nature [5], require a health-preserving interpretation of practical problems by a teacher. The competence-oriented aspect is that these problems have to be optimally solved by an educator in situ (lat. on the spot) and in a temporal "mode" hic et nunc (lat. now and at present). Accordingly, a teacher needs to be able to understand and interpret in a health-oriented manner the risks as well as to effectively manage them by way of active and purposeful implementation of health-preserving technologies, strategies and tactics. In the latest humanistic and nature-friendly, in essence, socio-cultural traditions, an appreciatively significant is the fact that now the diversity and multidimensionality of human nature are presented as an individual specificity and peculiarity. In our time of Eurocentric and humanistic transformations of the Ukrainian educational landscape, such visions and understanding of human nature are reflected in the introduction of inclusive culture and inclusive education [16].

In the scientific and pedagogical literature, the range of problems of development of health-preserving competence of a Physical Education teacher under conditions of postgraduate education based on comprehension of human nature through the methodological, technological and appreciative reflection of the knowledge of prevention of acute bronchitis [10], influenza, Covid-19 and acute respiratory diseases is insufficiently disclosed. Given the importance of the systemic and global impact of the Covid19 pandemic, seasonal "waves" of "ordinary" influenza and acute respiratory diseases on children's health under 
conditions of an educational process, we define this range of problems as relevant.

The aim of this study is the innovation-oriented improvement of the methodology, methods and practices for the development of health-preserving competence of a Physical Education teacher under conditions of postgraduate education based on the transfer of knowledge of the prevention of acute bronchitis, influenza, Covid-19 and acute respiratory diseases.

\section{Methods of the research}

The study used the following methods and approaches: the analysis of the scientific literature, innovative, competence $[17,18]$, systemic, problem, morphological and functional, phenomenological, axiological [19], philosophical, anthropological [5, 6, 19-21], ontological, epistemological, narrative, existential [4], temporal, synergetic [22, 23], pathopedagogical $[1,10]$, etiological (in health pedagogy) $[1,10]$, propaedeutic (in health pedagogy), narrative, existential, psychological, preventive [1, 10, 24], transdisciplinary [25, 26], hermeneutic [17] and inclusive [16].

Humanitarian, pedagogical and anthropological concepts and methods. The study used the following concepts: knowledge transfer [27], diffusion of innovations [28], humanisation of education [1-4], anthropologisation [4, 5, 19-21], "health-welfare" by H. Sigerist [29], homeostasis [30] and "methodological technologies" [31]. The methodological potential of maieutic, existential [4] and dialogic pedagogy was used. One of the central in this study was the use of systems of pedagogical tasks $[32,33]$.

The principles and approaches of continuing education [34], andragogy [35] and acmeology, as well as problembased learning [36], game methods [37], flipped learning [38], maieutic and dialogic methodologies, brainstorming technology [39], examination and analysis of the pedagogical best practice of teachers were used [40].

Paideia ideas. The ideas of the ancient Greek spiritual and educational system of Paideia (ancient Greek $\pi \alpha \iota \delta \epsilon \iota \alpha)$ were used [41, 42] as well as Hellenistic concepts of human nature (ancient Greek $\phi v \sigma \iota \zeta$ $\tau \mathrm{o} v \alpha v \theta \rho \omega \pi \mathrm{o} v$ ) [41], observance of measure (ancient Greek $\sigma v \mu \mu \epsilon \tau \rho \mathrm{o} v \mu \epsilon \tau \rho \iota \mathrm{o} v$ ) [41], harmony (ancient Greek $\kappa \rho \alpha \zeta$ ), healthy lifestyle, self knowledge (ancient Greek $\gamma v \omega \sigma \eta \alpha v \tau \mathrm{o} v$ ) (gnothi sautou) (interpreted by M. Foucault) $[43,44]$ and care of oneself (ancient Greek $\pi \iota \mu \lambda \iota \alpha$ $\alpha v \tau \mathrm{o} v$ ) (epimelēsthai sautou) (interpreted by M. Foucault) [43-45].

Digital technologies. Digital technologies were actively used - the work with Internet resources, in particular: augmented reality [46], virtual reality [47] and cloud technologies [48].

Our own methodological and procedural concepts. The system-organizing aspect of the research methodology is the "The disciplinary and methodological matrix of health-preserving competence of a Physical Education teacher" that was developed by us [1] and "Methodology of developing health-preserving competence of a Physical Education teacher based on the knowledge of prevention of acute bronchitis, influenza and acute respira- tory diseases". Based on the application of the idea of "integrated disciplines" (according to A. Subetto) [49], we have formed and applied such integrated disciplines as "Pathopedagogy" [1], "Propaedeutics of Health" [1] and "Health therapy" [1]. These "integrated disciplines" are considered as components and levels of the presented above disciplinary and methodological matrix. We used the dynamic health-preserving monitoring and healthpreserving protocol developed by us. The ideas of hierarchisation of health-preserving knowledge were used and health-preserving communication was actualised and applied.

Methods of performance control. The methodology is represented by the system consisting of 8 tasks and 10 questions. Its purpose is to control the knowledge, intellectual skills and readiness of a Physical Education teacher for health- preserving activity based on the knowledge of the prevention of acute bronchitis, influenza and acute respiratory diseases. This methodology is part of the "Methodology of developing health-preserving competence of a Physical Education teacher based on the knowledge of prevention of acute bronchitis, influenza and acute respiratory diseases". The tasks and questions are presented in a test form. A Physical Education teacher has to choose 1 correct answer out of 4 . Let us present a list of tasks and questions.

Tasks:

1. "Prevention of complications of acute rhinitis".

2. "Prevention of complications of bronchitis".

3. "Prevention of sudden cardiac death after influenza".

4. "Prevention of heart disorders due to the influence on respiration".

5. "Prevention of movement structure disorders (running) due to the influence on respiration".

6. "The influence of breath holding arreston the heart and respiratory system".

7. "The influence of nasal breathing during strenuous physical exercise on the cardiovascular and respiratory systems".

8. "The influence of hypothermia on the respiratory system and heart".

\section{Questions:}

1. What are the main disorders (in the sense of a disease) in the respiratory system that can occur due to influenza and how this process can affect motor activity?

2. What are the benefits of rinsing the nose with water and what are its possible consequences?

3. Is it advisable to purposefully and constantly train the respiratory system using mainly special breathing exercises? 
4. Cough and dyspnea indicate the possible disorders in the respiratory system and one more system. Which one?

5. Is it necessary to purposefully actualise the maximum involvement of all muscles that ensure the respiratory process during the motor activity?

6. At the expense of what structures and mechanisms are the inhalation and exhalations realized?

7. What is the health hazard of tonsillitis and how to take it into account when organizing physical activity?

8. How such a complication of bronchitis as pneumonia is dangerous for a human?

9. In which structures in bronchitis are the main pathological changes and how you can influence this process by means of physical culture?

10. In which structures are the main pathological changes in pneumonia and how you can influence this process by means of physical culture?

The "Results" section presents 5 tasks that were used for both performance monitoring and training (in a slightly modified and detailed format).

Methods of mathematical statistics. In order to compare the results of the study before and after the implementation of "The methodology of the development of healthpreserving competence of a Physical Education teacher based on the knowledge of prevention of acute bronchitis, influenza and acute respiratory diseases", in the experimental group, we used a Student's t-test [50]. Given the need to confirm the statistical significance of the difference between the mean values of the two dependent samples, the following algorithm of the Student's t-test was used:

1. We check the normality of the data distribution in the dependent samples, which are compared.

We calculate the empirical values of the skewness coefficient (skewness) (Skew) (1) and excess kurtosis $(K u r t)$ (2) for the dependent samples. The obtained values are compared with the corresponding critical values of skewness $\left(S k e w_{c r i t}\right)$ (3) and excess kurtosis $\left(\right.$ Kurt $\left._{\text {crit }}\right)$ (4).

$$
\text { Skew }=\frac{\sum_{i=1}^{n} z_{i}^{3}}{n},
$$

where:

$n$ is a sample size;

$z_{i}(i=1 \ldots n)$ is calculated by the following formula

$$
z_{i}=\frac{x_{i}-\bar{X}}{\sigma}
$$

$\sigma-$ the standard deviation is calculated by the following formula

$$
\sigma=\sqrt{\frac{\sum_{i=1}^{n}\left(x_{i}-\bar{X}\right)^{2}}{n-1}} ;
$$

$\bar{X}$ - expected value (arithmetic mean).

$$
\begin{gathered}
\text { Kurt }=\frac{\sum_{i=1}^{n} z_{i}^{4}}{n}-3 . \\
\text { Skew }_{\text {crit }}=3 \cdot \sqrt{\frac{6 \cdot(n-1)}{(n+1) \cdot(n+3)} .} \\
\text { Kurt }_{\text {crit }}=5 \cdot \sqrt{\frac{24 \cdot n \cdot(n-2) \cdot(n-3)}{(n+1)^{2} \cdot(n+3) \cdot(n+5)}} .
\end{gathered}
$$

If the empirical values of the skewness and excess kurtosis respectively are less than the critical values of the skewness and excess kurtosis, a decision is made on the normality of the data distribution.

2. We check the availability of a direct correlation between the dependent samples. We use Pearson correlation coefficient $r_{x_{1} x_{2}}$. For its application it is necessary for the data to be distributed in the normal way.

We formulate statistical hypotheses:

$H_{0}: r_{x_{1} x_{2}}=0$, no correlation between data;

$H_{1}: r_{x_{1} x_{2}}>>0, p<0.05$; there is a direct correlation between the data, which is significant at the level of 0.05 .

Pearson correlation coefficient $r_{x_{1} x_{2}}$ is calculated by formula (5):

$$
r_{x_{1} x_{2}}=\frac{n \sum_{i=1}^{n} x_{1 i} x_{2 i}-\sum_{i=1}^{n} x_{1 i} \cdot \sum_{i=1}^{n} x_{2 i}}{\sqrt{\left(n \sum_{i=1}^{n} x_{1 i}^{2}-\left(\sum_{i=1}^{n} x_{1 i}\right)^{2}\right) \cdot\left(n \sum_{i=1}^{n} x_{2 i}^{2}-\left(\sum_{i=1}^{n} x_{2 i}\right)^{2}\right)}}
$$

where $n$ is a sample size.

The calculated coefficient $r_{x_{1} x_{2}}$ is compared with the critical value $r_{\text {crit }}$ (according to the table of critical values of Pearson correlation coefficient). If $r_{x_{1} x_{2}}<r_{c r i t}$, then we accept hypothesis $H_{0}$ about the absence of correlation, and if $r_{x_{1} x_{2}} \geq r_{\text {crit }}$, then we accept hypothesis $H_{1}$ that there is a direct correlation between the data, which is significant at the level of $p<0.05$.

3. We calculate Student's t-test $(t)$ (6) for dependent samples $X_{1}$ and $X_{2}$. We compare the calculated empirical value of Student's t-test with the critical tabular value of Student's t-test. We come to the conclusion that hypothesis $H_{0}$ about the absence of differences is confirmed or rejected. 
Application of Student's t-test is carried out step-bystep:

a) We formulate statistical hypotheses:

$H_{0}$ : the differences between $X_{1}$ and $X_{2}$ are random and insignificant.

$H_{1}$ : the differences between $X_{1}$ and $X_{2}$ are trustworthy, significant.

b) We calculate the differences $d_{i}(i=1 \ldots n)$ between pairs of sample values $X_{1}$ and $X_{2}$.

c) We calculate the empirical value of Student's t-test $(t)$ by formula (6):

$$
t=\frac{\left|\bar{X}_{d}\right|}{\sigma_{d} / \sqrt{n}},
$$

where $\bar{X}_{d}$ is arithmetic mean of differences of pairs of values of $d_{i}(i=1 \ldots n)$;

$\sigma_{d}$ is standard deviation of differences of pairs of values of $d_{i}(i=1 \ldots n)$;

$n$ is a sample size;

d) We find the critical value of $t_{\text {crit }}$ in the table of critical values of Student's t-test, taking into account the number of degrees of freedom $d f=n-1$. If $t>t_{c r i t}$, we accept hypothesis $H_{1}$. If $t \leq t_{c r i t}$, we accept hypothesis $H_{0}$.

\section{Results and discussion}

The process of improving the health-preserving competence of a Physical Education teacher [1] under conditions of postgraduate education is realised through the use of the transfer of knowledge about the prevention of bronchitis, influenza and acute respiratory diseases [7, 10]. Practiceoriented knowledge of the morphophysiology of the respiratory system under normal conditions and in pathology as well as understanding of respiration as an anthropocultural phenomenon are also used as system-forming and "background" ones. Nowadays the knowledge about the prevention of COVID-19 is also used [11]. Besides, both the levels of methodology and methods as well as technologies and practices are actualised. At the level of methodology, the "Disciplinary and methodological matrix of healthpreserving competence of a Physical Education teacher" [1] that was developed by us is used as a competenceoriented, essential and system-organising methodological technologized strategy.

Methods, technologies and practices are implemented through the use of a system of interdependent and practiceoriented tasks $[32,33]$ and issues. The examination and analysis of situations, problems, educational and anthropological phenomena are performed both under normal conditions and in borderline states as well as in case of pathology.

Let us consider the methodological aspect presented by "The disciplinary and methodological matrix of healthpreserving competence of a Physical Education teacher"
[31]. This matrix is implemented on the basis of specific competence and innovation-oriented "methodological technology". The term "disciplinary and methodological matrix" was used by T. Kuhn [51, 52] who used it to denote the system of scientific ideas, schemes, intentions and attitudes dominant in a certain epoch. In this pedagogical system, a disciplinary and methodological matrix has a special, concretised, applied and specific purpose. The disciplinary and methodological matrix of health-preserving competence of a Physical Education teacher is developed on the basis of the use of systemic, special-purpose and competence approaches [17, 18]. In this aspect, the competency approach is a key one, within the framework of which the requests and needs for the design or improvement of a particular competence are the methodological intention and practical orientation that determine the system and direction of selection and configuration of certain disciplines, ideas, concepts, values that are used to perfect a specialist. Thus, the disciplinary and methodological matrix is formed primarily on the basis of requests aimed at solving specific educational and life problems as well as by technological and axiological comprehension of the phenomenon of human health and nature both under normal conditions and under the condition of crisis situations and pathology.

Within the framework of this matrix, the concepts, ideas, values, practices and technologies, which are aimed at solving a certain system of problems and are necessary for the improvement of the health discipline, are integrated. This approach is a prerequisite for the formation of a range of technological capabilities. It also contributes to the unlocking of epistemological, personal and axiological potential of a person, which is a necessary "competence condition" for optimal professional functioning of a Physical Education teacher. A similar methodological approach to training specialists exists in sociology and medicine. In particular, in medicine there has been a gradual transition from the study of disciplines that reveal human phenomenology in general and in normative formats to the coverage of human nature under conditions of pathology and by presenting specific anthropological phenomena, followed by a transition to practices and technologies. From a methodological standpoint, this matrix is formed as a "movement" along the "epistemological axes": "fundamentalisation - technologisation", "from general to specific"; "from problems to phenomena"; "from knowledge, schemes and ideas to the cognition of phenomena in reality"; "from knowledge to practice-oriented knowledge and practices and technologies"; "from the general values of human to technological values that determine activity"; "from the idea to learn and know to - to navigate, act and be able to". The disciplinary and methodological matrix of health-preserving competence of a Physical Education teacher (see figure 1) [1] is a "dynamic epistemological system", in which six qualitatively different levels are singled out. The learning process is organised on the basis of interaction and transfer of knowledge, values and meanings among different levels. Therefore, this system is not only linearly consistent (although this aspect is present in it) but also contains feedback. The specified 
multilevel structure determines the possibilities for forming in an educator the capacity for conceptualisation based on the practice-oriented actualisation of the whole spectrum of knowledge about a human. Let us consider the six-level structure of this matrix:

Level I - General ideas of a human

Level II - Pathopedagogy

Level III - Propaedeutics of health

Level IV - Health therapy

Level V - Health-preserving practices

Level VI - Health-preserving technologies

This disciplinary and methodological matrix uses an epistemological idea of health-oriented understanding and disclosure of human nature and his/her health through systemic, transdisciplinary [25], problem, selective and at the same time special-purpose, purposeful and practiceoriented cognition. This matrix is developed on the basis of competence paradigm [17, 18], ideas of praxeology, practical interpretations of T. Kuhn's ideas [51, 52], receptions of the concept of "Methodological technology" by B. Sazonov and H. Shchedrovytskyi (work "Methodology as technology") [31], application of O. Subetto's [49] concept of "integrated disciplines" that are developed on the basis of educational requests up to the formation of a certain competence.

We represent this disciplinary and methodological matrix as a methodological technology for the development of a health-preserving competence of a Physical Education teacher. In the content and semantic as well as the axiological and motivational framework of this matrix we will present some fragments of the content part as an example of an application of this methodological technology. Accordingly, we will resolve some issues of the phenomenology of the respiratory system under normal conditions and conditions of pathology using the transfer of knowledge about the prevention of bronchitis [7, 10], influenza and acute respiratory diseases.

Level I-General ideas of a human is a system of disciplines, scientific areas of research, ideas, technologies and practices that form mainly theoretical and partly technological and, at the same time, professionally oriented "general idea" about a human as a multidimensional phenomenon. This is realised by studying relevant and generalised issues of health preservation based on the knowledge of such disciplines as anatomy, histology, physiology, sports physiology, ecology, anthropology (physical, psychological, environmental, social, sports, philosophical), methodology, pedagogy, philosophy, psychology and others.

At the above-mentioned level, attention is selectively focused on the technologically significant knowledge [10] of the respiratory system under normal conditions. As an example of the content component of this pedagogical system we will briefly present some system-organising knowledge. Of fundamental importance for a teacher's understanding of the risks of the formation of respiratory disorders is not the details of the structure, but the fact that the conductive and functional parts are singled out in the respiratory system. The conductive part includes the nasal cavity, pharynx, larynx, bronchial trachea and bronchioles. The conductive part as a whole has the general plan of the structure presented by the tubular formations covered with a stratified squamous or pseudostratified (a variant of a single-layer) ciliated epithelium with the inclusion of goblet cells producing mucus. The above-mentioned formation $[7,10]$ structurally determines the peculiarities of "smooth" and "slow" (in comparison with pneumonia) course of inflammation and the tendency to transition of acute bronchitis (that is formed relatively quickly - from several hours to 3-6 months) to a chronic form (lasting for a long time - month and years). A typical disorder in this case is bronchitis.

The functional part (in which the process of respiration is realised) is represented by acini (literal translation of "bunches of grapes"), which are represented by a singlelayer epithelium that can be easily and quickly damaged. Gas exchange takes place in acini. Acini, which consist of alveoli, can be damaged quickly and therefore inflammation or their other damage proceeds relatively quickly, with a pronounced acute phase and is not transformed into a chronic (long or continuously proceeding) form. This disorder is called pneumonia.

When revealing the phenomenology of the respiratory system at level (I) in the process of improving the level of professional skills, it is practically important issues, phenomena, situations and tasks that are selectively actualised. It is through the consideration of these issues, situations and tasks that the repetition and restoration of general ideas about the respiratory system take place. Accordingly, the orientation and specification (concretisation) of knowledge about the respiratory system is carried out for the purpose of applying them in the healthpreserving practices and technologies.

Level II - Pathopedagogy [1] is formed by the disciplines that aim to reveal the phenomenology of a human under conditions of stress, tension, pathology and extreme conditions. This is realised by studying such disciplines as pathological physiology, pathological anatomy, clinical physiology, stressology, defectology, ecology and inclusion. The above-mentioned areas are integrated by us in the format of the "integrated discipline" [49] Pathopedagogy.

At this level, we actualise knowledge about general and particular pathological processes [1,24]. This knowledge is basic and conceptualising, and, accordingly, forms the basis of the ticular, for the purpose of understanding of pr health-preserving thinking of a teacher. Relevant and essential in this aspect is the doctrine of the causes of the disorders - the etiology and mechanisms of disease development - pathogenesis. The basis of prevention is based on knowledge of the causes. And the prevention of complications of bronchitis is formed on the basis of general ideas about the mechanisms of disease development pathogenesis. Therefore, it is consciously and intellectu- 


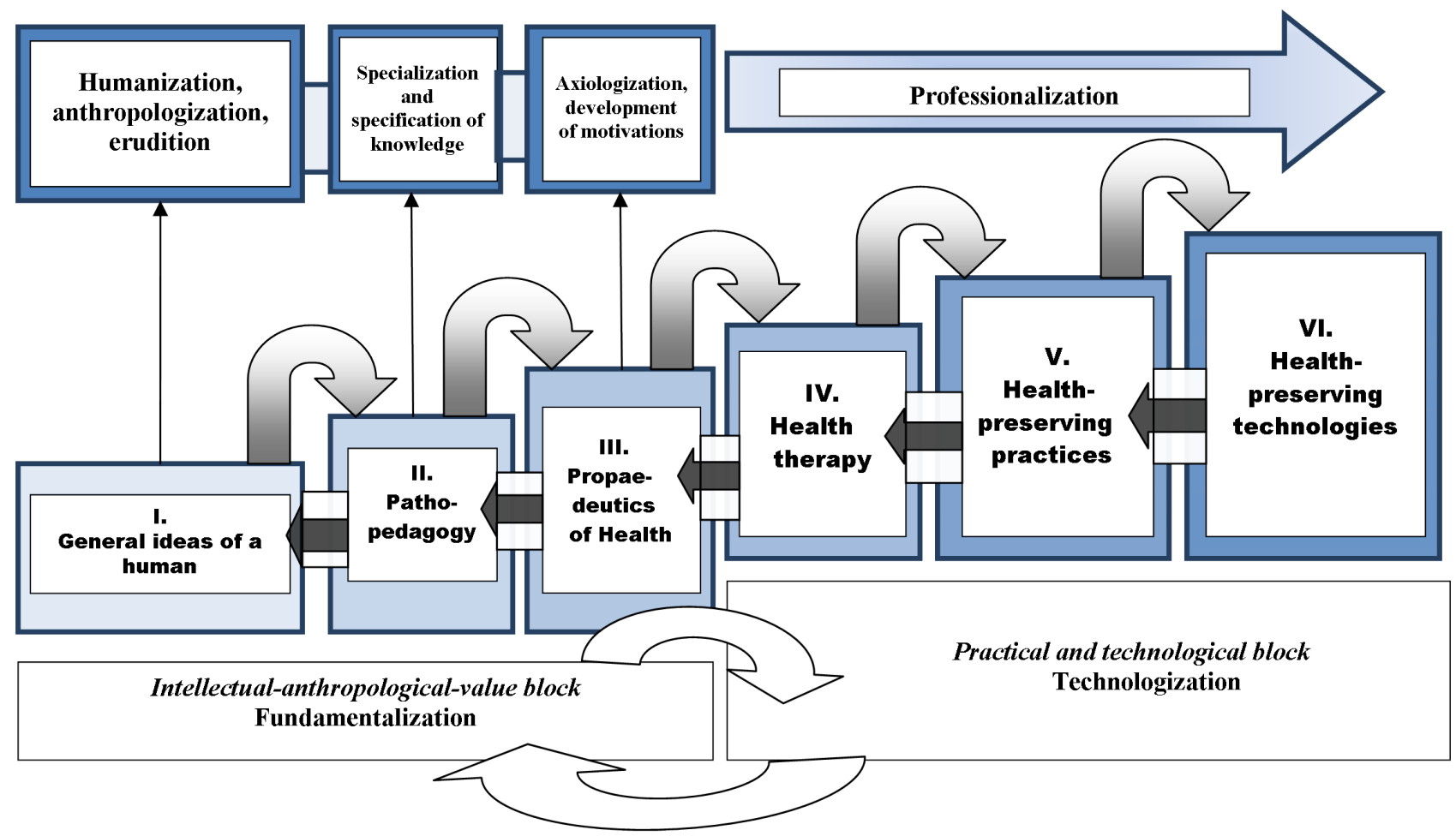

Figure 1. Scheme of the structure of the disciplinary and methodological matrix of health-preserving competence of a physical education teacher. The arrows show the transfer of knowledge

ally, and not only on the basis of prescription that prevention is formed using the knowledge of the causes and some ideas about the pathogenesis.

In particular, for the purpose of understanding of prevention by a teacher, we briefly mention the phenomenon of inflammation that is divided into three stages, each of which lasts 7 days. In reality, we mainly observe and realise the problem for health when the second stage of inflammation is manifested - exudation (accumulation of the fluid). For example, for diagnostics, it is important to understand that this stage of exudation (accumulation of the fluid) is manifested (i.e. we can observe it and ask about it) in the form of cough and shortness of breath. When the exudative stage of inflammation approaches its end, it is manifested, first of all, in the appearance of sputum when coughing. A relevant conclusion for practice is the strategy of complete exclusion of purposeful training at the exudation stage. If this is not done, then the inflammation will spread through the respiratory system, increasing the risk of complications.

In the process of improving the level of professional skills, the etiology (the science of the causes of pathologies) of acute bronchitis is examined in detail. Among the main causes of acute bronchitis, we can indicate infection (influenza, COVID-19, acute respiratory diseases and ornithosis that is spread by birds); temperature (mainly hypothermia); high humidity of the air and the presence in the inhaled air of irritants, toxic substances, allergens, microbes and dust [7, 9, 10].

Particular attention is paid to the issues of prevention of bronchitis complications, especially pneumonia as a central and essential problem, which is a systemic risk to the life of a child. In the context of axiological understanding of this range of problems, we point to the need for teachers to form, first of all, life-saving attitudes, intentions, values and meanings of prevention of acute bronchitis. This is especially important under conditions of the COVID-19 [9, 11] pandemic because this form of influenza is developing swiftly, "in a galloping manner", "avalanche-like" and leads to serious complications, and for some time, maybe asymptomatic (i.e. without manifestations) and then "appear" in all its manifestations. This necessitates a teacher's attention to the swift and latent (hidden) course of COVID-19 as its dangerous peculiarities.

The above-mentioned level contributes to the formation of the health-preserving thinking and appropriate intellectual strategies and tactics of a Physical Education teacher. A relevant aspect is the actualisation of responsibility for the life and health of students as a systemorganising professional value. When considering this range of problems, it is the life-saving aspect that becomes the main focus. An important area is also the prevention of bronchial asthma [8] and chronic bronchitis as probable complications of acute bronchitis. The above-mentioned prevention and correction can to some extent be implemented by means of physical culture.

Level III - Propaedeutics of health (anthropologically and axiologically-oriented propaedeutic level) [1], aimed at forming general ideas about really existing problems of a human and his/her health both under normal conditions and under conditions of possible pathology, including the 
knowledge of potential risks. Within the content and semantic framework of this level, the range of problems of professional ethics, values, personal qualities, standards and stereotypes of behaviour, intentions and professional attitudes is actualised, that is, those components that form the personal and existential as well as partially active and discursive components of health-preserving competence of a Physical Education teacher.

The problems of prevention are considered on the basis of the analysis and integration of the knowledge and values covered when considering levels I and II. Practice-oriented approaches to primary before-doctor diagnostics of the acute disorders of the respiratory system are formed. First of all, for example, attention is focused on the symptom of cough as an essential one for further health-preserving strategies.

In the context of the implementation of risk management strategies, the ways of implementing healthpreserving dynamic monitoring, singling out of risk groups and targeted working with them are presented. Issues of effective health-preserving communication under conditions of an educational process are actualised. At this level, a teacher learns: to communicate with children on the topic of prevention of acute bronchitis, to psychologically and organisationally influence them, to form and use preventive and corrective strategies, to observe and diagnose in general (before-doctor diagnostics) possible acute inflammatory pathology of the respiratory system.

Level IV - Health therapy (technologically and axiologically-oriented level of health therapy) [1] is aimed at practically and axiologically-oriented coverage of the phenomenology of a human and his/her health, risks and conditions of formation of certain pathologies and peculiarities of crisis, pedagogical and life situations that are dangerous to the life and health of a child. Statistically significant pathologies [7-11] and conditions that precede them are also studied in a practice-oriented way as well as general ideas about before-doctor diagnostics, strategies of providing help, prevention and correction with the possible use of means of physical culture are revealed.

At this level, in general, the following is analysed: peculiarities and differences of bronchitis caused by influenza, COVID-19 [9] and acute respiratory diseases; individual, sexual and developmental peculiarities of children's morphophysiology in the context of bronchitis prevention; strategies for the use of physical culture means to prevent such complications of bronchitis as bronchial asthma [8] and chronic bronchitis. Primary and special attention is paid to the focus on individual preventive and corrective work with students based on the study of peculiarities of their lives and problems. To do this, we use the diagnostic technology developed by us that is represented by a health-preserving protocol. This protocol reflects in dynamics (in time) both the positive state of a student's respiratory system, his/her health in general and some possible problems and pathologies considered in the context of and in relation to motor activity. That is, at this level, the knowledge is further specified (concretised), provided practical orientation, transformed into strategies and algorithms of action and is revealed as an intellectual tool for working with a particular person and specific problems and phenomena.

Level V-Health-preserving practices, including "bodily and motor" as well as "motor and intellectual" in their essence anthropopractices "self-practice" or "selftechnology" (according to M. Foucault) [44] that are implemented in an educational process and during extracurricular activities. Practices of diagnostics, correction and prevention of disorders of the respiratory system are also actualised. The conditions, approaches and ways of forming a safe and healthy educational environment are determined. That is, the level of practices determines the real educational process and, accordingly, in its essence, it is what forms a health-preserving environment. The ways of using, combining and integrating various educational technologies, methodologies, techniques, strategies and recommendations within the framework of educational practice and environment are analysed.

Level VI - Health-preserving technologies that also contains methodologies, recommendations, strategies, tactics and teaching techniques of preserving and forming health including diagnostic, corrective, developmental and preventive ones. At this level, a teacher acquires and tests certain health-preserving technologies and methodologies and correlates them with motor activity. Within the framework of using the potential of this level, it is important to have special teacher training, which is necessary for the proper implementation of certain health technologies and their selection as well as not using what is not evidential and may create certain health risks.

Health-preserving technologies are analysed from the standpoint of their suitability and possible adaptation and modification for use in acute bronchitis. Desemanticisation and demythologisation of typical myths and fixed notions of the preservation of respiratory health are carried out. For example, the "traditional" recommendations in acute bronchitis and other pathologies of the respiratory system such as to actively use physical exercise, including breathing exercises as well as conditioning to the cold temperatures are critically analysed.

Representing the technological potential of the level of health-preserving technologies, for example, we recommend relatively short and slow walking (300-800 m) in influenza and bronchitis, depending on the general condition. This recommendation is relevant only under favourable environmental and temperature conditions and in relatively good health. It is recommended to use "locally" and individually the elements of the respiratory gymnastics according to the system of K. Buteiko [53] for the purpose of "calming" breathing and "removing" bronchospasm.

Let us consider the key aspects of "The methodology of forming health-preserving competence of a Physical Education teacher based on the knowledge of prevention of acute bronchitis, influenza and acute respiratory diseases" [7, 9, 10]. The methodology is formed and implemented on the basis of the competence approach and "The disciplinary and methodological matrix of healthpreserving competence of a Physical Education teacher" [1]. The methodology is developed on the basis of use 
of pedagogical tasks [32,33], analysis and examination of issues, situations and anthropological phenomena (in particular, anatomy, physiology and pathology of the respiratory system and their cultural and professional interpretations and representations) [53] and representation of practical recommendations of strategies and tactics. In addition, the following are applied: the principles and ideas of acmeology [34, 35], problem-based learning, game methods, flipped learning, maieutic and dialogic methodologies, brainstorming technology, examination and analysis of the pedagogical best practice of teachers [40], illustrations and video fragments in which this range of problems is revealed; dynamic health-preserving observation is taught and the ways of using the health-preserving protocol are covered. Considerable attention is paid to the analysis and examination of certain motor modes, physical exercise, sporting and health-improving technologies, practical recommendations in the context of prevention of bronchitis, influenza and acute respiratory diseases $[7,9,10]$ as well as the correction of their consequences by means of physical culture. Nowadays the COVID-19 range of problems is being actualised $[9,11]$. Narrative, existential, ontological, temporal and transdisciplinary [25] approaches and archetypal psychology as well as the ideas of self-knowledge are used, and the use of the tradition of self-care is actualised [41, 43-45]. Within the framework of the application of the idea of self-knowledge [44], the teachers are recommended to test various exercises, methodologies and technologies of physical culture and health preservation by way of "testing" them first on themselves. It is also required for their professional, appropriate and optimal application or selection. Both during the period before the quarantine and at present, within the framework of the above-mentioned methodology, digital technologies are used, in particular, the work with the Internet resources, including augmented reality, virtual reality, distance learning (under conditions of quarantine) and cloud technologies.

This methodology is formed using the idea of hierarchisation of knowledge, meanings, values and strategies of health preservation. This is realised on the basis of hierarchisation of the importance of certain knowledge, skills, meanings and values as well as personal qualities for the purpose of optimising, professionalising and technologising preservation of health and life. A similar approach exists in medicine (but mostly without a formal singling out of levels). Besides, it corresponds to human nature, in which values, meanings and to some extent knowledge are hierarchical. This approach contributes to the development of such skills of a teacher: to actualise, conceptualise, problematise and foresee (anticipation aspect) complex situations as well as to act in an optimal way, rationally, technologically, quickly, using established schemes, algorithms of technological values and meanings and on the basis of singling out central and most serious problems. Accordingly, we single out 3 levels (see figure 2):

A - (one) - "Knowledge and skills in preserving life". Metaphorically, this is "Human Life". These are knowledge and skills that, first of all, concern life preservation and to some extent health. In this educational system, the greatest attention is paid to the knowledge of level A.

B - (two) - "Knowledge and skills in preserving health". Metaphorically, this is "Human Health". These are knowledge and skills that, first of all, concern health preservation and formation as well as adapting to the environment.

$\mathrm{C}$ - (three) - "Knowledge and skills relating to health formation and development". Metaphorically, this is "Human development and he/she is in the world". These are knowledge and skills that, first of all, are aimed at the comparable development of health and fitness. They also aim at personal development, the realisation of sustainable development goals, sociocultural and professional adaptation and inclusion.

A fragment of "The methodology of developing health-preserving competence of a Physical Education teacher based on the knowledge of prevention of acute bronchitis, influenza and acute respiratory diseases" is presented (in a somewhat abridged version) by 5 pedagogical tasks. These tasks are used both for training and controlling the level of knowledge. The presented variant of tasks is used to control knowledge, intellectual skills, strategies and values of health preservation and, in general, the readiness of a teacher for the realisation of health-preserving activity.

A variant of tasks, which is used for training, reveals the problem in a more extensive and detailed way and is also discussion oriented. When considering such a task in class, the problem revealed in it is analysed quite widely, using the gnosiological and activity potential embedded in the disciplinary and methodological matrix of the competence as well as using problem-based, systemic, existential, narrative and maieutic approaches. Not only separate tasks are analysed, but the systems of tasks and questions as well as strategies, values and cognitive schemes aimed at preserving health which are formed on their basis. The use of pedagogical tasks and analysis of certain pedagogical situations and anthropological phenomena are central to this methodology.

To control the knowledge, intellectual skills and readiness of a teacher to implement a health-preserving activity, pedagogical tasks and questions were used which were formed in the format of test tasks. Let us present a fragment of such a case of problems. It consists of five tasks. In total, 8 tasks were used. In addition, we used the questions presented as test tasks (10 in number). In addition to determining the level of knowledge, the use of the tasks was aimed at deepening the practice oriented knowledge in this area and forming health-preserving thinking, intentionality, goal setting and motivation of a teacher by "immersion" into a purely practical range of problems. Thus, the purpose of applying pedagogical tasks and as a whole of this methodology was the development of: cognitive (intellectual and axiological), activity and discursive as 


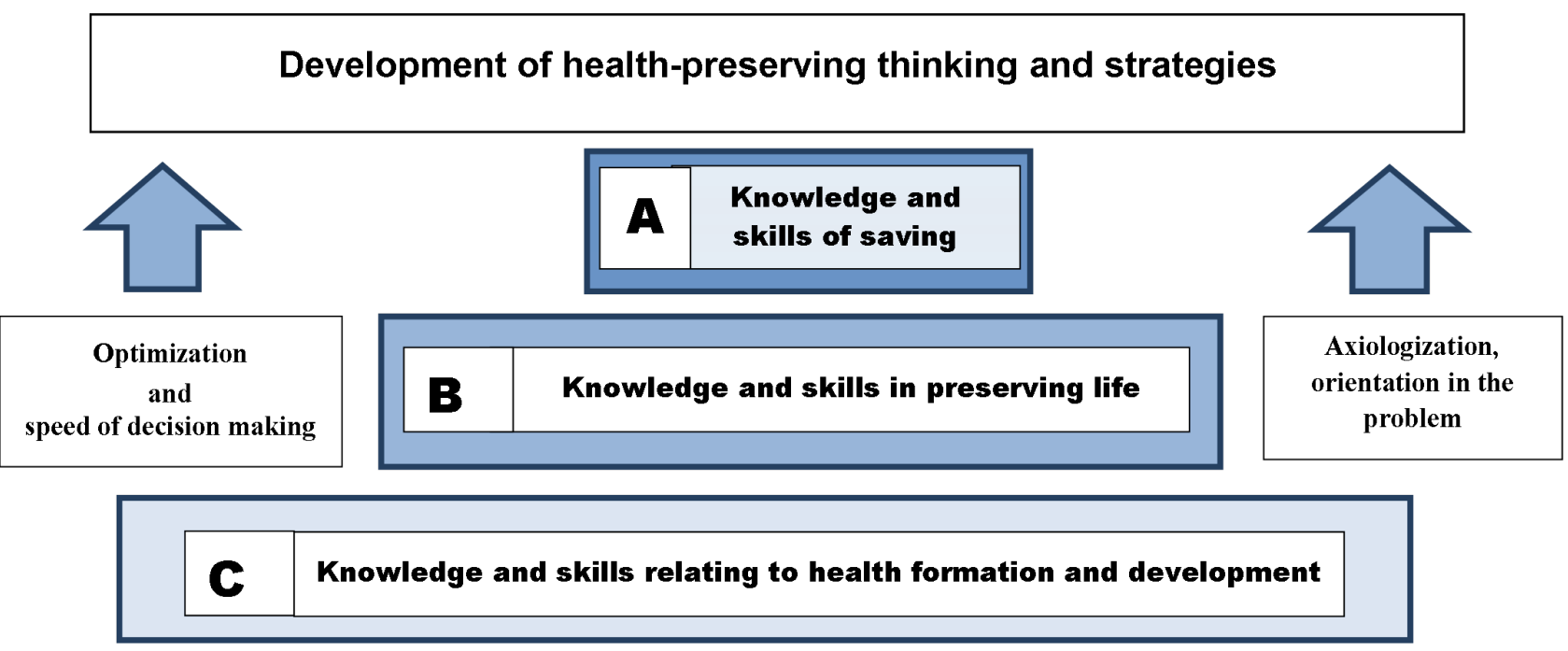

Figure 2. Schematic representation of the hierarchy of knowledge, meaning, values and health strategies. There are three levels - A, B, C.

well as personal and existential components of the healthpreserving competence of a Physical Education teacher. This paper mainly considers the development of the cognitive component based on the transfer of special knowledge about bronchitis and influenza, and the ideas, values and meanings of health preservation as well as by way of actualisation of the comparable intentions and cognitive and professional guidelines. Thus, the purpose of application of pedagogical tasks and this methodology as a whole was the development of: cognitive (intellectual and axiological), activity and discursive as well as personal and existential components of health-preserving competence of a Physical Education teacher. This paper mainly considers the issues of the development of the cognitive component based on the transfer of special knowledge about bronchitis and influenza and ideas, values and meanings of health preservation as well as by way of actualisation of the comparable intentions and cognitive and professional guidelines. The examples of the tasks are given below. The title of the task reflects its practical health-preserving essence.

The students were recommended to complete a pedagogical task by choosing a correct comment to a recommendation.

Task No. 1 - "Prevention of complications of acute rhinitis".

Recommendation. If in contrast to a relatively good general condition and health, a child has: general weakness combined with the active secretion of mucus from the nose, that is, there are signs of acute inflammation of the nose (rhinitis), then to preserve health and strengthen the immune system the following is recommended: deep breathing as well as physical exercise and breathing exercises which include deep and intense breathing.

Comment (answer):

1. Physical exercise and motor modes, the structure which includes deep and intense breathing in acute inflammation of the nose (rhinitis), contribute to the increase in oxygen in the blood, improve blood cir- culation in the lungs, cleanse the respiratory system and body, which in total contributes to rapid recovery. In influenza and acute respiratory diseases, this will speed a patient's rapid recovery and prevent complications.

2. Strenuous physical exercise and deep breathing in acute rhinitis will not only significantly increase the risks and accelerate the occurrence of complications such as acute bronchitis (inflammation of bronchial tubes) and, in some cases, pneumonia. In addition, breathing exercises in acute rhinitis do not affect the immune system in any way, but only contributes to health problems. In influenza and to a much lesser extent in acute respiratory diseases, this will increase the likelihood of severe form of the disease and the risk to life.

3. Strenuous physical exercise and deep breathing in acute rhinitis neutrally affect health without improving or worsening the condition.

4. Without strenuous physical exercise and deep breathing in acute rhinitis, the recovery is significantly complicated. These very methods are the basis for the prevention and treatment of acute rhinitis using before-doctor means. At the same time, immunity significantly improves, which is especially important in influenza and acute respiratory diseases.

Task No. 2 - "Prevention of complications of bronchitis”.

Recommendation. If a child has a cough, then deep breathing, physical exercise and breathing exercises that include deep and intense breathing are recommended to preserve good health and strengthen the immune system.

Comment (answer):

1. Physical exercise and motor modes, the structure which includes deep and intense breathing, when 
coughing that is mainly a sign of bronchitis, contribute to the increase in oxygen in the blood, improve blood circulation in the lungs, cleanse the respiratory system and body and improve immunity, which in total contributes to rapid recovery. In influenza and acute respiratory diseases, this will contribute to a quicker recovery.

2. If you have a cough, that is mainly a sign of bronchitis, strenuous physical exercise and deep breathing will lead to the spread of infection throughout the lungs and increase inflammation. Accordingly, this will significantly increase the risks and accelerate the occurrence of such life-threatening complications of bronchitis as pneumonia. Besides, deep breathing in this case does not affect the immune system in any way, but only contributes to health problems. In influenza and to a lesser extent in acute respiratory diseases, this will increase the likelihood of severe form of the disease and lead to a substantial increase in life-threatening risks.

3. Strenuous physical exercise and deep breathing in bronchitis neutrally affect health without improving or worsening the condition.

4. Without strenuous physical exercise and deep breathing in bronchitis, the recovery is significantly complicated. These very methods are the basis for the prevention and correction of bronchitis using before-doctor means. Besides, immunity significantly improves, which is especially important in bronchitis caused by influenza and acute respiratory diseases.

Task No. 3 - "Prevention of sudden cardiac death after influenza".

Recommendation. Strenuous physical exercise including breathing exercises is recommended after influenza. Such a relatively intense training process will lead to the cleansing of the lungs and body from waste products and its rapid rehabilitation and recovery.

Comment (answer):

1. Relatively strenuous physical exercise and deep breathing after influenza improve cardiac performance, which in total contributes to rapid rehabilitation and recovery. In influenza, it will contribute to a quicker recovery.

2. Strenuous physical exercise and deep breathing after the previous influenza neutrally affect health without improving or worsening the condition.

3. Without strenuous physical exercise and deep breathing after the previous influenza, the recovery is significantly complicated. These very methods are the basis for the recovery of the cardiovascular system and body as a whole after influenza or after acute respiratory diseases. This significantly improves immunity, which is especially important for the recovery of the cardiovascular system and body after influenza.
4. Relatively strenuous physical exercise and deep breathing after the previous influenza will significantly increase the risk of emergence of acute heart diseases, in particular, sudden cardiac death. In influenza, this will increase the likelihood of acute cardiac pathology, in particular, sudden cardiac death, and lead to a substantial increase in lifethreatening risks. This is due to the fact that the influenza virus, in addition to the bronchial mucous tunic, affects the microcirculatory bloodstream (the smallest vessels of the body) of the whole body, including such vital organs as the heart, brain and kidneys.

Task No. 4 - "Prevention of heart disorders due to the influence on respiration".

Recommendation. Which of the recommendations aimed at synchronising the steps with breathing while running is optimal and "more" physiological (appropriate to human nature): "During the run, one inhalation is recommended for two steps and one exhalation for three steps or vice versa (one inhalation for three steps and one exhalation for two)". Another variant of an answer is possible. Comment on your answer using modern physiological and psychophysiological notions.

Comment (answer):

1. While running, it is better to inhale for three steps and exhale for two. Breathing control will contribute to the proper organisation of physiological and mental processes and indicate a developed will and consciousness of a person. It will also contribute to health improvement. During influenza and acute bronchitis, the above-mentioned control over the respiratory function will significantly improve health.

2. The most acceptable and physiological is almost complete absence of conscious and purposeful synchronisation of breathing and running. During the run, the synchronisation process is automatic and unconscious. Conscious and relatively long-term control of respiration and the attempts to synchronise it with movement will lead to desynchronisation of the respiratory and cardiac systems, which will increase the risk of acute cardiac pathology, including sudden cardiac death. In influenza and acute bronchitis, this control of the respiratory function will significantly increase the risks to life and health.

3. While running, it is better to inhale for two steps and exhale for three. Breathing control will contribute to the proper organisation of physiological and mental processes and indicate a developed will and consciousness of a person. It will also contribute to health improvement. During influenza and acute bronchitis, this control over the respiratory function will significantly improve health.

4. During the run, the presence of "total" control over breathing or its absence will not affect health in any way, including cardiac one. 
Task No. 5 - "Prevention of movement structure disorders (running) due to the influence on respiration".

Recommendation. Which of the recommendations aimed at synchronising the steps with breathing during the run is optimal and "more" physiological (appropriate to human nature): "During the run, one inhalation is recommended for two steps and one exhalation for three steps or vice versa (one inhalation for three steps and one exhalation for two)", which will contribute to the improvement of a training process and corresponding rise in results. The other answer is possible. Comment on your answer using modern physiological and psychophysiological notions.

Comment (answer):

1. While running, it is better to inhale for three steps and exhale for two. Breathing control will contribute to the proper organisation of physiological and mental processes and indicate a developed will and consciousness of a person. It will also contribute to the improvement of movement structure and its coordination and, in general, will increase the efficiency of a training process. In addition, during influenza and acute bronchitis, this control over the respiratory function will significantly improve health.

2. While running, it is better to inhale for two steps exhale for three. Breathing control will contribute to the proper organisation of physiological and mental processes and indicate a developed will and consciousness of a person. It will also contribute to the improvement of movement structure and its coordination and, in general, will increase the efficiency of a training process.

3. During the run, the presence of "total" control over breathing or its absence will not affect the structure of movement (running) in any way.

4. The most acceptable and physiological (in the sense of appropriate to nature) is almost complete absence of conscious and purposeful synchronisation of breathing and running. Besides, the synchronisation process is partially automatic. This is due to the fact that while running, the respiratory function, according to its physiological and semantic significance, is secondary towards motor activity. That is, running is not formed on the basis of breathing goals. On the contrary, breathing provides the realisation of running. At the same time, respiration is the primary functional system that ensures running (according to P. Anokhin's doctrine of functional systems) of type one.

The specified functional system, based on the innate internal automatic mechanisms, adapts to motor activity "on its own". Conscious control over breathing and the attempts to synchronise it with the movement will disrupt the entire structure of running and turning it into a "breathing-running" exercise. A runner will change the goal of running as a process of moving in space. This "spatial and motor goal" will be transformed into the goal

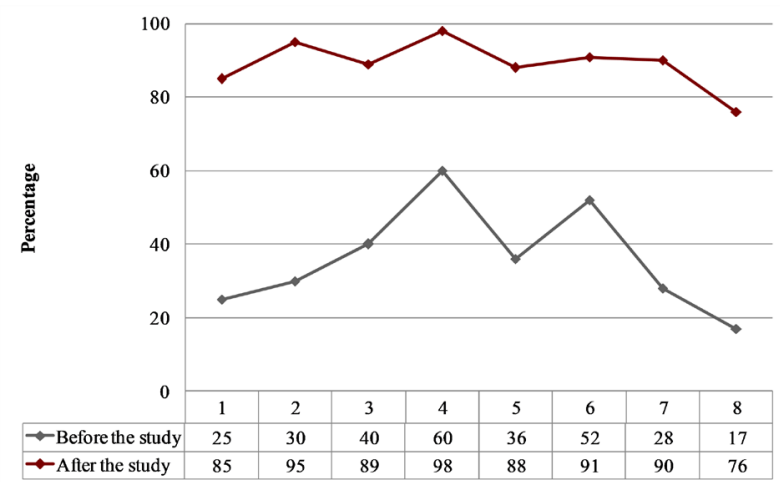

Figure 3. The results showing the number of correct answers of Physical Education teachers to the tasks before and after the implementation of "The methodology of the development of healthpreserving competence of a Physical Education teacher based on the knowledge of prevention of acute bronchitis, influenza and acute respiratory diseases".

of control over the physiological process - over breathing. This will be realized in the person himself/herself - in his/her psyche and in physiology. That is, the traditional structure of goals and meanings characteristic of running is destroyed. During influenza and acute bronchitis, this control over respiratory function will significantly increase the risk of emergence of acute cardiac pathology.

Experimental study. The experimental study, which was conducted in 2017-2018, involved 816 Physical Education teachers. The experimental group included 411 people. The study was conducted at 9 higher educational institutions in Ukraine: Drohobych Ivan Franko State Pedagogical University, Chernihiv Regional Institute of Postgraduate Pedagogical Education named after K. D. Ushinsky, Sumy Regional Institute of Postgraduate Pedagogical Education, Mykolaiv Regional Institute for Postgraduate Pedagogical Education, Zaporizhzhia Regional Institute of Continuing Pedagogical Education, Donetsk Regional Institute of Postgraduate Teacher Education, Zhytomyr Regional Institute of Postgraduate Pedagogical Education, Kherson Academy of Continuing Education, Lviv Regional Institute of Postgraduate Pedagogical Education.

The analysis of the results of the study showed that after the introduction of the methodology of developing health-preserving competence of a Physical Education teacher based on the knowledge of prevention of acute bronchitis, influenza and acute respiratory diseases, in the experimental group there was a positive dynamics of academic achievements of Physical Education teachers (see figure 3, figure 4). The figures show the dynamics of the survey results of Physical Education teachers before and after the introduction of this methodology. Figures show the percentages of correct answers to the questions of 8 problems and 10 tests respectively.

Let us prove the statistical confidentiality of the obtained results. The number of tasks that had to be completed by the Physical Education teachers before and after the implementation of the methodology of development of health-preserving competence of Physical Edu- 


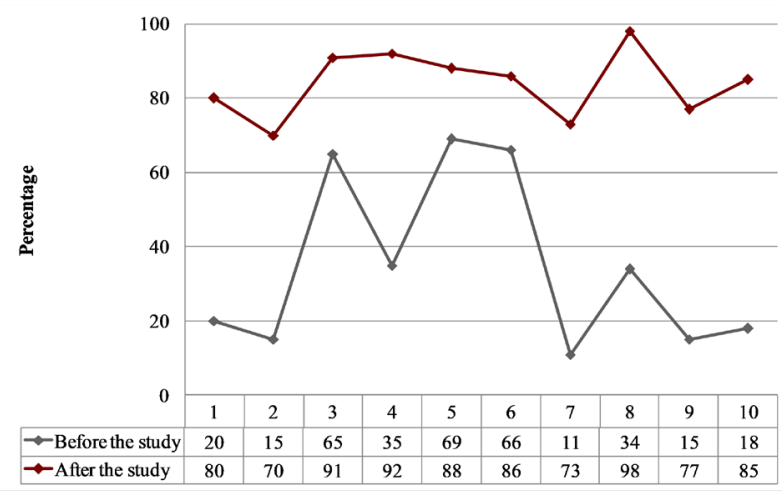

Figure 4. The results showing the number of correct answers of Physical Education teachers to the questions of the tests before and after the introduction of "The methodology of the development of health-preserving competence of a Physical Education teacher based on the knowledge of prevention of acute bronchitis, influenza and acute respiratory diseases".

cation teachers based on the knowledge of prevention of acute bronchitis, influenza and acute respiratory diseases $n_{1}=18$ and $n_{2}=18$.

Let us confirm the statistical significance of the difference between the mean values of the two samples before and after the implementation of this methodology in the experimental group with the help of Student's t-test [50]:

1. We check the normality of the data distribution in samples $X_{1}$ and $X_{2}$ before and after the implementation of the methodology respectively.

The empirical values of skewness calculated by formula (1) in samples $X_{1}$ and $X_{2}$ are equal to $S k e w_{1} \approx 0.5, S k e w_{2} \approx-0.43$ respectively. The critical value of skewness, calculated by formula (3) is equal to $S k e w_{\text {crit }} \approx 1.52$. We compare the empirical values of skewness with the critical one. $S k e w_{1} \approx 0.5<S k e w_{\text {crit }} \approx 1.52$, $\mid$ Kew $_{2}|\approx|-0,43 \mid<S$ kew $_{\text {crit }} \approx 1.52$.

The empirical values of excess kurtosis in samples $X_{1}$ and $X_{2}$ calculated by formula (2) are equal to Kurt $_{1} \approx-1.26$, Kurt $_{2} \approx-0.91$ respectively. The critical value of excess kurtosis calculated by formula (4) is equal to Kurt $_{\text {crit }} \approx 3.86$. We compare the empirical values of excess kurtosis with the critical one. $\mid$ Kurt $_{1}|\approx|-1.26 \mid<$ Kurt $_{\text {crit }} \approx 3.86$. $\mid$ Kurt $_{2}|\approx|-0.91 \mid<$ Kurt $_{\text {crit }} \approx 3.86$.

Since the empirical values of skewness and excess kurtosis for both samples $X_{1}$ and $X_{2}$ respectively are less than the critical values of skewness and excess kurtosis, we make a decision that the data in samples $X_{1}$ and $X_{2}$ are distributed according to the normal distribution law.

2. We check the availability of a direct correlation between samples $X_{1}$ and $X_{2}$. Since the sample data $X_{1}$ and $X_{2}$ are distributed according to the normal distribution law, we can use the Pearson correlation coefficient $r_{x_{1} x_{2}}$.
We formulate statistical hypotheses:

$H_{0}: r_{x_{1} x_{2}}=0$, no correlation between sample data $X_{1}$ and $X_{2}$;

$H_{1}: r_{x_{1} x_{2}}>>0, p<0.05$; there is a direct correlation between the sample data $X_{1}$ and $X_{2}$, which is significant at the level of 0.05 .

The Pearson correlation coefficient $r_{x y}$ is calculated by formula $(5), r_{x_{1} x_{2}} \approx 0.61$.

According to the table of critical values Pearson correlation coefficient, significance level $p<0.05$, $r_{\text {crit }}=0.47$. Therefore, $r_{x_{1} x_{2}} \approx 0.61 \geq r_{\text {crit }}=0.47$ - we accept hypothesis $H_{1}$ that there is a direct correlation between the data of samples $X_{1}$ and $X_{2}$, significant at the level of $p<0.05$.

3. Since the data of samples $X_{1}$ and $X_{2}$ are distributed according to the normal law and there is a direct correlation between them, in this case we can use Student's t-test to compare these samples. We formulate statistical hypotheses:

$H_{0}$ : the differences between $X_{1}$ and $X_{2}$ are random and insignificant;

$H_{1}$ : the differences between $X_{1}$ and $X_{2}$ are trustworthy and significant.

The empirical value of Student's t-test for samples $X_{1}$ and $X_{2}$ is calculated by formula $(6) t \approx 13.75$.

Critical value in the table of critical values of Student's t-test with the degrees of freedom $d f=18-1=17, t_{\text {crit }}=2.11 ; t \approx 13.75>t_{\text {crit }}=2.11$, we accept hypothesis $H_{1}$.

Therefore, we conclude that the differences between $X_{1}$ and $X_{2}$ are trustworthy and significant. That is, differences in the percentage of correct answers of Physical Education teachers to the questions and tests before and after the introduction of the methodology of developing healthpreserving competence of a Physical Education teacher based on the knowledge of prevention of acute bronchitis, influenza and acute respiratory diseases are significant.

\section{Conclusion}

As a holistic professional and personal, activity and cognitive phenomenon, the health-preserving competence of a Physical Education teacher is aimed at forming a healthy lifestyle and meaningful and purposeful risk management. This includes, above all, the prevention and correction of major problems, conditions and pathologies. One of the central problems represented in the format of prevention and correction of pathology is acute bronchitis that occurs as a manifestation of influenza, in particular, COVID-19 and acute respiratory diseases.

For a Physical Education teacher, this range of problems has both health-preserving and life-saving significance. The presence of the COVID-19 pandemic dramatically increases the risk of life-threatening conditions and pathologies, especially those that can be triggered by physical exercise. The relevance and professional significance 
of knowledge for a Physical Education teacher are determined by the factors of prevention of acute bronchitis, influenza, COVID-19 and acute respiratory diseases.

The essential methodological strategy for improving health-preserving competence of a Physical Education teacher under conditions of the postgraduate education that is presented by "The Disciplinary and methodological matrix of health-preserving competence of a Physical Education teacher". This matrix is a six-level epistemological system in which knowledge is transferred among different levels. In the end, this leads to a practice oriented direction of knowledge about human nature as well as to their specification (concretisation), technological and axiological orientation. The matrix is formed according to the system and target principles and is aimed at professionalisation, methodologisation, technologisation, anthropologisation, axiologisation of health-preserving competence of a Physical Education teacher as well as knowledge about health and educational practices and technologies of health preservation of participants of an educational process. The system of the above-mentioned matrix uses the following developed by us "integrated disciplines": "Pathopedagogy", "Health Propaedeutics" and "Health Therapy", which are both equal and sequential stages aimed at the development of all components of health-preserving competence. Due to the application of this matrix and the developed "integrated disciplines", the knowledge about a human both under normal conditions and under conditions of stress and pathology is integrated, axiologised and anthropologised being included in the structure of healthpreserving thinking and also becoming a cognitive and axiological basis of values, meanings, intentions, actions, and strategies as well as improvement and manifestation of professionally oriented psychological and characterological qualities.

A methodological basis of educational and methodological activity is presented by 'Methodology of developing health-preserving competence of a Physical Education teacher based on the nnowledge of prevention of acute bronchitis, influenza and acute respiratory diseases". The methodology is based on the competency approach, "The disciplinary and methodological matrix of healthpreserving competence of a Physical Education teacher", problem-based and flipped learning, andragogy, game, maieutic and dialogic methods as well as narrative, existential, anthropological, humanistic approaches, as well as the ideas of self-knowledge, the Hellenistic concept of "self-care" (according to M. Foucault) and others.

Central to the methodology is the use of pedagogical tasks, as well as the analysis, examination and interpretation of complex pedagogical and life situations and issues. The use of pedagogical tasks and analysis of situations of this methodology as a whole is aimed at improving: mainly cognitive (intellectual and axiological), as well as for the development of activity and discursive, personal and existential components of health-preserving competence of a Physical Education teacher.

Within the framework of this methodology, the knowledge, values and practical strategies for maintaining health, which are formed on the basis of them, are hier- archised with the singling out of three levels: A - (one) "Knowledge and skills of preserving life", B - (two) "Knowledge and skills in forming health and development", C - (three) "Knowledge and skills about health formation and development". Level A is obligatory for full understanding and practical use; levels B and C are less significant so they are given less attention.

The analysis of the results of the study using Student's t-test showed the effectiveness of the implementation of "The methodology of developing health-preserving competence of a Physical Education teacher based on the knowledge of prevention of acute bronchitis, influenza and acute respiratory diseases". It was established that the level of academic performance of Physical Education teachers in the experimental group after the introduction of this method was significantly higher than the level of academic performance of Physical Education teachers before the research. First of all, this concerns the first 5 tasks related to level A, which is obligatory for full acquisition. A significant gap in the knowledge between the initial level (before the training) and after the training is explained by the novelty of this knowledge and area in general, practical significance and relative interest of the teachers, the use of the hierarchy of knowledge as an effective teaching technique owing to which the technology oriented meanings, strategies and axiological attitude to solving problems of prevention of bronchitis and influenza as well as correction of their consequences are formed by means of physical culture.

The presented methodology and methods, as well as approaches, visions and directions aimed at developing health-preserving competence of a Physical Education teacher and preserving health under conditions of an educational process, are correlated with the classical idea of "health as a good" (in essence, an interpretation of an ancient Greek concept of agate) of H. Sigerist. These methodologies and methods are humanistic, ecocentric and those that are consistent with the spirit, ideology and sustainable development of goals, among which the preservation of health and motor activity are presented as essential and system-organizing.

\section{References}

[1] V.M. Fedorets, Visnyk Zhytomyrskoho derzhavnoho universytetu imeni Ivana Franka. Pedahohichni nauky 4, 220 (2018)

[2] O. Elkin, L. Hrynevych, S. Kalashnikova, P. Khobzey, I. Kobernyk, V. Kovtunets, O. Makarenko, O. Malakhova, T. Nanayeva, R. Shiyan et al., The New Ukrainian School. Conceptual principles of secondary school reform) (Ministry of Education and Science of Ukraine, Kyiv, 2016), https://mon.gov.ua/storage/app/ media/zagalna\%20serednya/Book-ENG.pdf

[3] M.V. Nabok, Dukhovnist osobystosti: metodolohiia, teoriia i praktyka 3, 159 (2017)

[4] I. Bostad, in Enlightenment, Creativity and Education: Polities, Politics, Performances, edited by 
L. Wikander, C. Gustafsson, U. Riis (Brill Sense, 2012), pp. 45-61, ISBN 978-9462090507

[5] K. Ushinskii, Chelovek kak predmet vospitaniia. Opyt pedagogicheskoi antropologii (Man as a subject of education. An attempt at pedagogical anthropology), Vol. 8 (Izdatelstvo Akademii pedagogicheskih nauk RSFSR, Moscow, Leningrad, 1950), https://imwerden.de/pdf/ushinsky_ sobranie_sochineny_tom08_1950_text.pdf

[6] H. Zdarzil, Padagogische Anthropologie (Educational anthropology) (Quelle und Meyer, New York, 1972)

[7] G.A. Samsygina, Pediatrics 2, 25 (2008)

[8] D.P. Strachan, B.K. Butland, H.R. Anderson, BMJ 312, 1195 (1996)

[9] M. Belingheri, M.E. Paladino, M.A. Riva, Occupational Medicine pp. 82-83 (2020)

[10] V.M. Fedorets, Pedahohichnyi almanakh 25, 224 (2015)

[11] F.M. Reimers, A. Schleicher, A framework to guide an education response to the COVID-19 Pandemic of 2020 (OECD, Paris, 2020)

[12] O. Burov, A. Kiv, S. Semerikov, A. Striuk, M. Striuk, L. Kolgatina, I. Oliinyk, CEUR Workshop Proceedings 2731, 1 (2020)

[13] K. Polhun, T. Kramarenko, M. Maloivan, A. Tomilina, Journal of Physics: Conference Series 1840, 012053 (2021)

[14] N.S. Ponomareva, Journal of Physics: Conference Series 1840, 012035 (2021)

[15] M. Velykodna, Psychodynamic Practice 27, 10 (2021)

[16] A. Kolupaieva, O. Taranchenko, Inclusive education: from basics to practice (Inclusive education: from basics to practice) (ATOPOL, Kyiv, 2016)

[17] D. Raven, O.N. Yaryigin, A.A. Korostelev, Azimut nauchnyih issledovaniy: pedagogika i psihologiya $\mathbf{6}$, 167 (1997)

[18] S. Hodge, T. Mavin, S. Kearns, Vocations and Learning 13, 27 (2019)

[19] T.A. Jumanovich, T.D. Eshboevna, European Journal of Research and Reflection in Educational Sciences 7, 702 (2019)

[20] S.D. Blum, American Anthropologist 121, 641 (2019)

[21] M.N. Nevzorov, Ph.D. thesis, Khabarovskiy gosudarstvennyy pedagogicheskiy universitet, Khabarovsk (1999)

[22] V.H. Kremen, Ridna shkola 6, 3 (2010)

[23] V. Soloviev, N. Moiseienko, O. Tarasova, Communications in Computer and Information Science 1175 CCIS, 231 (2020)

[24] O. Klochko, V. Fedorets, O. Maliar, V. Hnatuyk, 166, 10033 (2020)

[25] M. Slavinec, B. Aberšek, D. Gačević, A. Flogie, Journal of Baltic Science Education 18, 435 (2019)

[26] L. Bilousova, L. Gryzun, D. Sherstiuk, E. Shmeltser, CEUR Workshop Proceedings 2433, 336 (2019)
[27] H. Takeuchi, I. Nonaka, Hitotsubashi on knowledge management (Wiley, 2004)

[28] E.M. Rogers, Diffusion of innovations (Simon and Schuster, New York, 2010)

[29] H.E. Sigerist, Medicine and human welfare (Yale University Press New Haven, 1941)

[30] C. Bernard, Introduction à l'étude de la médecine expérimentale (J. B. Baillière et Fils, Paris, 1865), https://www.irphe.fr/ $\sim$ clanet/otherpaperfile/articles/Bernard/ bernard_introduction_etude_medecine_ experimentale.pdf

[31] B. Sazonov, Metodologiya kak tekhnologiya (Methodology as technology), in Arkhiv Chteniy pamyati G. P. Shchedrovitskogo: Materialy XI Chteniy (Archive of the Readings in memory of $G$. P. Shchedrovitsky: Materials of the XI Readings) (2005), https://www . fondgp.ru/old/lib/ chteniya/xi/mat/8.html

[32] C. Herodotou, M. Sharples, M. Gaved, A. KukulskaHulme, B. Rienties, E. Scanlon, D. Whitelock, Innovative pedagogies of the future: An evidencebased selection, in Frontiers in Education (Frontiers, 2019), Vol. 4, p. 113

[33] N.O. Diachenko, Ph.D. thesis, Instytut vyshchoi osvity NAPN Ukrainy, Kyiv (2015)

[34] I.E. Rüber, S.L. Rees, B. Schmidt-Hertha, International Review of Education 64, 543 (2018)

[35] V. Arghode, E.W. Brieger, G.N. McLean, European Journal of Training and Development 41, 593 (2017)

[36] K. Vlasenko, V. Achkan, O. Chumak, I. Lovianova, T. Armash, Universal Journal of Educational Research 8, 2853 (2020)

[37] T. Vakaliuk, V. Kontsedailo, D. Antoniuk, O. Korotun, I. Mintii, A. Pikilnyak, CEUR Workshop Proceedings 2547, 66 (2020)

[38] A. Abdula, H. Baluta, N. Kozachenko, D. Kassim, CEUR Workshop Proceedings 2643, 306 (2020)

[39] N. Balyk, I. Grod, Y. Vasylenko, V. Oleksiuk, Y. Rogovchenko, Journal of Physics: Conference Series 1840, 012032 (2021)

[40] I. Han, W.S. Shin, Y. Ko, Teachers and Teaching 23, 829 (2017)

[41] W. Jaeger, Pajdejya. Vospitanie antichnogo greka (Paideia. The education of the ancient Greek) (Greko-latin. kabinet Yu. A. Shichalina, Moskva, 1997)

[42] M. Ibrahimov, Visnyk Zhytomyrskoho derzhavnoho universytetu imeni Ivana Franka 1, 9 (2014)

[43] M. Foucault, The History of Sexuality, Vol. 3: The Care of the Self (Vintage, 1998)

[44] M. Foucault, Technologies of the Self: Lectures at University of Vermont Oct. 1982 (University of Massachusets Press, Cambridge, 1988)

[45] I.S. Kuznetsova, Mnogoobraziye fiksatsiy kontsepta "zabota o sebe" v obrazovanii (Variety of fixations of the concept of "self-care" in education), in "Zabota o sebe" kak obrazovatelnaya praktika sovre- 
mennogo klassicheskogo universiteta: sb. st. i materialov mezhdunar. nauch. konf. (24-25 noyab. 2018) (2018)

[46] P. Nechypurenko, T. Starova, T. Selivanova, A. Tomilina, A. Uchitel, CEUR Workshop Proceedings 2257, 15 (2018)

[47] S. Symonenko, N. Zaitseva, V. Osadchyi, K. Osadcha, E. Shmeltser, CEUR Workshop Proceedings 2547, 37 (2020)

[48] O.M. Markova, S.O. Semerikov, A.M. Striuk, Information Technologies and Learning Tools 46, 29 (2015)

[49] A. Subetto, Teoriya fundamentalizatsii obrazovaniya $i$ universalnyye kompetentsii (noosfernaya paradigma universalizma) (The theory of fundamentalization of education and universal competences (noospheric paradigm of universalism)) (Asterion, St. Petersburg, 2010)

[50] Student, Biometrika 6, 1 (1908)

[51] T. Kuhn, The structure of scientific revolutions, 1st edn. (Univ. Chicago, Chicago, 1962)

[52] A.P. Ogurtsov, Distsiplinarnaya struktura nauki; eye genezis i obosnovaniye (Disciplinary structure of science; its genesis and rationale) (Nauka, Moskva, 1988)

[53] K.P. Buteyko, V.K. Buteyko, M.M. Buteyko, Strogoye izlozheniye osnov teorii K. P. Buteyko o fiziologicheskoy roli dykhaniya $v$ genezise nekotorykh zabolevaniy (A rigorous presentation of the foundations of the K. P. Buteyko theory on the physiological role of respiration in the genesis of some diseases) (Obshchestvo Buteyko, 2005) 